\title{
Efficacy and Safety of Perioperative Bridging Anticoagulation in Patients with Atrial Fibrillation Undergoing Elective Surgical Procedures: A Meta-analysis
}

\author{
Muhammad Umer Siddiqui, MD; Ahmed K. Pasha, MD; Ibtisam Rauf, MD; Justin Z. Lee, MD; \\ Muhammad Danial Siddiqui, MD; Youssef Yaacoub, MD; and Mohammad Reza Movahed, MD, FACC, PhD
}

Objective: The study objective was to determine if peri-operative bridging anticoagulation in patients with atrial fibrillation is beneficial or harmful.

Design: Systematic review and meta-analysis.

Setting: Inpatient or in-hospital setting.

Participants: Adults with atrial fibrillation having a CHADS2 score >I undergoing elective surgical procedure on anticoagulation.

Methods: A systemic search of multiple databases (Cochrane, Medline, PubMed) was performed regarding studies conducted on efficacy and safety of perioperative bridging anticoagulation in patients with atrial fibrillation. Studies identified were reviewed by two authors individually before inclusion. The results were then pooled using Review Manager to determine the combined effect. Stroke/systemic embolism was considered as the primary efficacy outcome. Major bleeding was the primary safety outcome.

Results: The systematic search revealed 108 potential articles. The full texts of 28 articles were retrieved for assessment of eligibility. After full text review, 25 articles were excluded. Three articles met inclusion criteria. No significant difference in stroke/systemic embolism with bridging anticoagulation was noted (risk ratio, I.25-95\% confidence interval [Cl], 0.55-2.85). Bridging was associated with significantly higher risk of major bleeding (risk ratio, 3.29-95\% Cl, 2.25-4.8I).

Conclusion: An individualized approach is required when initiating peri-operative bridging anticoagulation. There is certainly a higher risk of bleeding with bridging anticoagulation and no difference in stroke/systemic embolism. However, the results cannot be extrapolated to patients who have valvular atrial fibrillation or CHADS2 score of 5 or greater.

Keywords: Bridging; Atrial fibrillation; Perioperative anticoagulation; Stroke; Systemic embolism and bleeding

A trial fibrillation (AF) is a rising health concern, being the most common arrhythmia, ${ }^{1}$ and its prevalence is expected to be 5.6 million by the year 2050 in the United States. ${ }^{2}$ Oral anticoagulation therapy (OAC) is used to reduce the risk of stroke and other thromboembolic events. In patients with AF on OAC who have to undergo surgical/ invasive procedures, OAC therapy must be held temporarily, which may expose patients to a higher risk of thromboembolic
Corresponding Author: Muhammad Umer Siddiqui, MD, Marshfield Clinic 1700 W Stout St., Rice Lake, WI, 54868, Tel: (7I5) 65I-96I2, Email: siddiqui.muhammad@marshfieldclinic.org
Received: December 16, 2020

Ist Revision: March 26, 2020

2nd Revision: September 8, 2020

Accepted: September II, 2020 
events. During this period, parenteral anticoagulation in the form of low molecular weight heparin or unfractionated heparin may be used to reduce the risk of thromboembolic events, although, this bridging therapy has its own risks and benefits. ${ }^{3}$ In clinical practice, health care professionals encounter this situation quite frequently. There are few guidelines available in this regard, ${ }^{4,5}$ and there still appears to be a lack of evidence. Siengal et $\mathrm{al}^{6}$ discussed the role of bridging anticoagulation, but their study included patients not only with AF but with other indications for anticoagulation including mechanical heart valves, venous thromboembolism, and thrombotic states. ${ }^{6}$ We present a meta-analysis of randomized and observational studies to assess evidence for safety and efficacy of perioperative bridging in patients with AF.

The objectives of this study were to determine the efficacy and safety of peri-operative anticoagulation with anticoagulation in adults with AF having a $\mathrm{CHADS}_{2}$ of $>1$ undergoing elective surgical procedure when compared to a similar population not undergoing peri-operative bridging. Only one study included $\mathrm{CHA}_{2} \mathrm{DS}_{2}$ Vasc Score. 7

\section{Methods}

Data Sources and Search Strategy

This systematic review and meta-analysis was reported according to Preferred Reporting Items for Systematic Review and Meta-Analyses (PRISMA) guidelines. ${ }^{8}$ A systematic search of Medline (Ovid), Cochrane Central Register for Controlled Trials, and PubMed was performed from database inception through January 2019. Initial keywords utilized to perform searches were atrial fibrillation; stroke; anticoagulation; perioperative bridging; bridging; and elective surgical procedure. Medical terms (MeSH) search bar was used to identify keywords available in Medline, PubMed, and Cochrane registry. They were then combined using AND/OR. To search for grey literature and unpublished articles, we used the aforementioned keywords to perform a search in www. clinicaltrials.gov. Once the search was performed, the citations were exported to EndNote X7.5 (Thompson ISI ResearchSoft, Philadelphia, Pennsylvania) Reference Manager and duplicates were removed.

\section{Study Selection and Data Extraction}

Two authors (MUS, AKP) independently screened citations revealed after the search. If there were any disagreements, a third review author (JL) was asked to arbitrate. Studies were included if they comprised adults with AF having a $\mathrm{CHADS}_{2}$ score of $>1$ undergoing an elective surgical procedure on vitamin $\mathrm{K}$ antagonist or non-vitamin $\mathrm{K}$ antagonist oral anticoagulants (NOAC). Studies that included patients admitted for emergent surgery, having a mechanical heart valve, or stroke/systemic embolism/transient ischemic attack (TIA) within 12 weeks were excluded, as this patient population is considered high risk for perioperative thromboembolism. ${ }^{5}$
A data collection form was created in Microsoft word and was shared among the review authors. Data regarding study characteristics, intervention, comparison, and outcomes were documented on the form (Supplementary Tables 1-3, available online). Two authors (MUS, AKP) independently extracted outcome data on a standardized data extraction tool. Any disagreements were resolved by discussion. One author (MUS) transferred data into Review Manager (RevMan) version 5.3 (The Cochrane Collaboration 2014, Nordic Cochrane Centre Copenhagen, Denmark).

\section{Outcomes}

The primary intervention was perioperative bridging anticoagulation in patients with AF undergoing elective surgery. Arterial thromboembolism (stroke, systemic embolism, or TIA) was considered as the primary efficacy outcome. Major bleeding (gastrointestinal bleeding, intracranial bleeding, bleeding from other sites) was the primary safety outcome.

\section{Risk of Bias}

Risk of bias for each study was assessed by two authors independently using the criteria outlined in the Cochrane Handbook for Systematic Reviews of Interventions. ${ }^{9}$ Any disagreements were resolved by discussion. For randomized control trials (RCTs), Cochrane risk of bias tools for RCTs was utilized (Supplementary Table 4, available online). For cohort studies, Scottish Intercollegiate Guidelines Network (SIGN) methodology was utilized to assess the risk of bias (Supplementary Table 5, available online). Each potential source of bias was graded as high, low, or unclear.

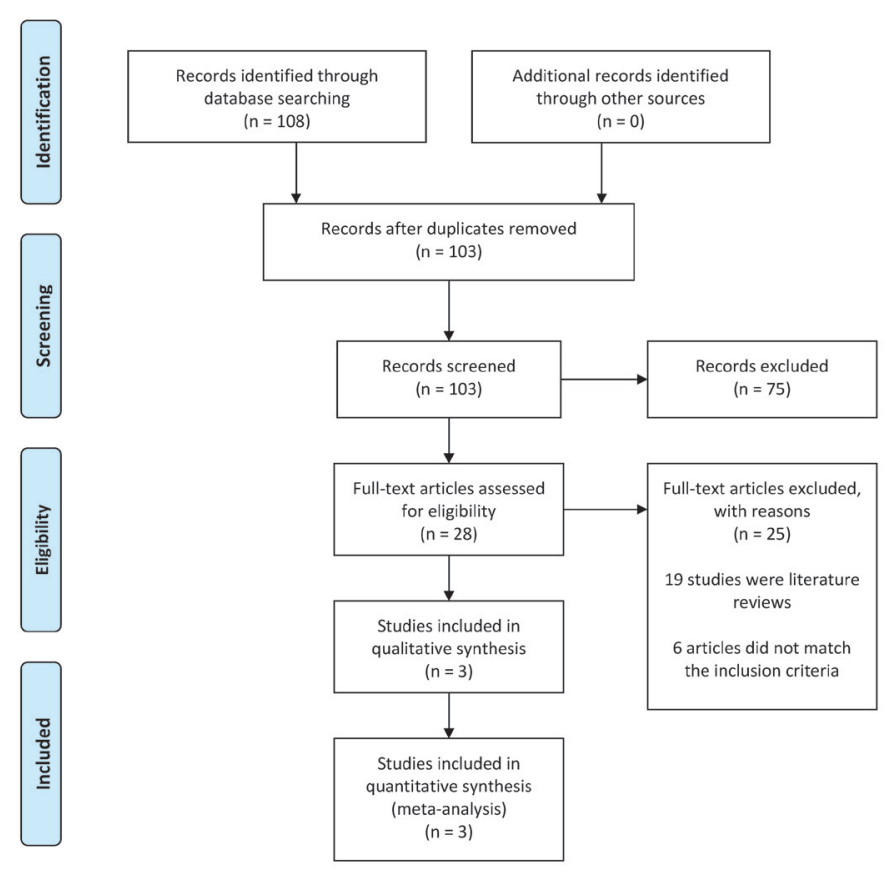

Figure 1. PRISMA flow diagram 
Statistical Analysis

The statistical analyses were undertaken using Review Manager (RevMan). Outcomes from each study were pooled and compared using a random-effects (RE) model to adjust for between study variance. The treatment effect was reported as odds ratio (OR) and 95\% confidence interval (CI). The $\mathrm{I}^{2}$-statistic was quantified to measure heterogeneity with values $>25 \%, 50 \%$, and $75 \%$ consistent with low, moderate, and high degrees of heterogeneity, respectively. ${ }^{10}$ Statistical significance was reached when $P$ values were $<.05$. To further adjust the heterogeneity between the studies, the inverse variance heterogeneity (IVhet) estimator was implemented using MetaXL version 5.3. When compared to random effects model estimator, IVhet model has shown a lower observed variance regardless of heterogeneity.

\section{Results}

\section{Baseline Demographics}

After the search, 108 potential articles were found. A PRISMA flow diagram (Figure 1) is included for details. After removal of duplicates, 103 articles were screened. There were 75 studies not pertaining to bridging anticoagulation that were excluded after initial screening. The full texts of 28 articles were retrieved for assessment of eligibility, and 25 articles were excluded after full-text review. Among the excluded 25 articles, 19 studies were review articles, 2 studies included patients with mechanical valves, and 4 studies looked at the safety and efficacy of anticoagulation in patients undergoing radiofrequency catheter ablation of AF. There were three articles selected to perform the systematic review and meta-analysis., ${ }^{711,12}$

This review identified 1,899 patients who were provided perioperative bridging anticoagulation, and 4,406 patients who did

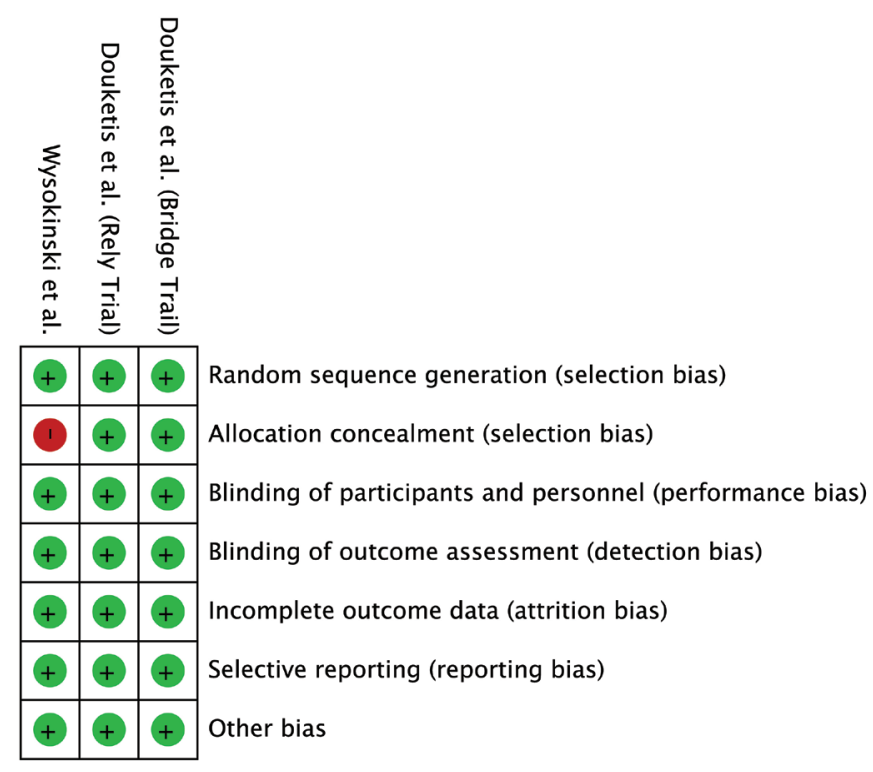

Figure 2. Risk of bias summary not receive peri-operative anticoagulation. Among the three studies, two were randomized controlled trials, ${ }^{7,11}$ and one was an observational study. ${ }^{12}$ Two studies were published in 2015, 711 and one study was published in 2008..$^{12}$ All three trials were conducted in the United States, and the baseline characteristics were consistent in all three. The mean age of the participants in the bridging arm ranged between 71.1 and 73.0 years, whereas the mean age range in the non-bridging arm was 71.8 and 74 years. The mean $\mathrm{CHADS}_{2}$ score was 2.25 in the bridging arm and 2.1 in the non-bridging arm. Warfarin was the OAC used by the selected population in two studies, but one study included patients both on warfarin and dabigatran. ${ }^{7}$ All studies used low molecular weight heparin and heparin for bridging anticoagulation.

A risk of bias evaluation was performed using the Cochrane risk of bias tool (Supplementary Table 4, available online) for randomized trials ${ }^{7,11}$ and the SIGN tool (Supplementary Table 5, available online) for observational studies. ${ }^{12}$ All three studies reported a low risk of bias overall (Figure 2). The participants, personnel, and outcomes assessment were blinded in all studies, suggesting low risk of performance and detection bias. Similarly, incomplete outcome data or selective reporting were not observed in the three included studies, decreasing the risk of attrition and reporting bias. The risk of selection bias was noted to be high in the study conducted by Wysokinski et al, ${ }^{12}$ as the method of allocation concealment was not reported.

\section{Arterial Thromboembolism}

When comparing bridging anticoagulation with no bridging in patients with AF undergoing an elective surgical procedure, all three included studies in the meta-analysis individually did not show any statistically significant difference in risk of stroke and systemic embolism.

Pooled result of these three studies showed no difference in reducing stroke and/or systemic embolism when comparing bridging anticoagulation with no-bridging (OR 1.25 [0.552.85], $P=.60$; Figure 3 ). The result did not differ when we excluded the subgroup of patients in the Douketis et al ${ }^{7}$ RELY trial sub-study who were on dabigatran for perioperative anticoagulation instead of warfarin (OR 1.09 [0.42 - 2.85], $P=.86$; Figure 4). No variation was noted between the trials as indicated by low $\mathrm{I}^{2}$ value of $0 \%$.

The pooled result of the three studies using IVhet model was similar to RE model and showed no difference in reducing stroke and/or systemic embolism when comparing bridging anticoagulation with no bridging (OR 1.25 [0.55-2.85]; Supplementary Figure 1, available online).

\section{Major Bleeding}

When reviewed individually, one study ${ }^{12}$ did not show any statistically significant difference in major bleeding, whereas the other two studies ${ }^{7,11}$ showed significantly increased risk of major bleeding favoring the no bridging arm. Pooled result of 


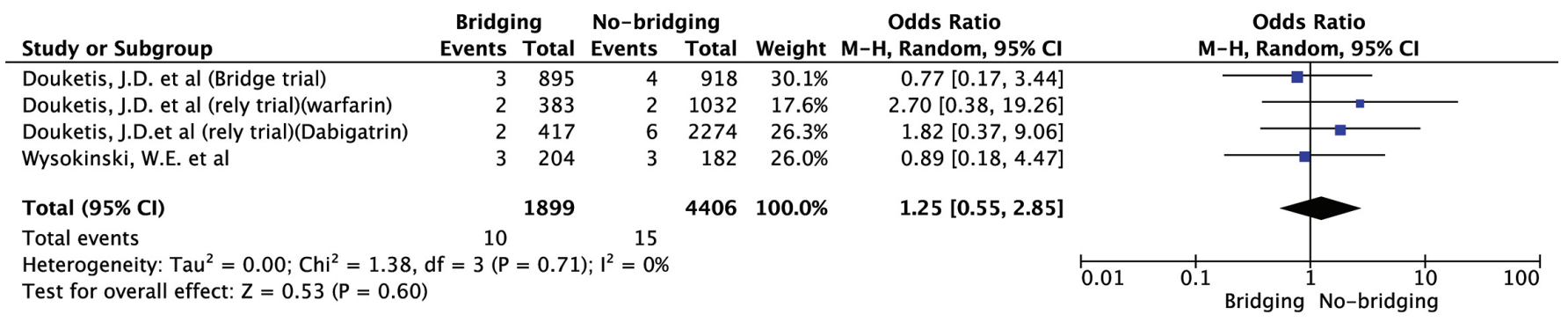

Figure 3. Forest plot for primary efficacy outcome

these three studies identified significant difference in major bleeding, favoring no bridging when compared to bridging anticoagulation (OR 3.29 [2.25-4.81], $P<.001$; Figure 5). The safety outcome did not differ when we excluded patients on dabigatran for perioperative anticoagulation in the RELY trial ${ }^{7}$ (OR 2.92 [1.58-5.40], $P<.001$; Figure 6). Low and moderate variation was noted between the trials as indicated by $\mathrm{I}^{2}$ value of $20 \%$ and $43 \%$, respectively. When analyzed using the IVhet model, the pooled result of the three studies was similar to the RE model and showed significantly higher major bleeding in the bridging anticoagulation group (OR 3.32 [2.28-4.84]; Supplementary Figure 2, available online).

\section{Discussion}

We reviewed three studies, but there were two oral anticoagulants (warfarin and dabigatran) used in the study by Douketis et al, ${ }^{7}$ hence, the analysis was performed on four subgroups/studies. The total number of patients who had their bleeding and thromboembolic risk assessed exceeded 6,000. Of these, 4,406 patients were in the no bridging arm, and 1,899 patients were in bridging arm. The study denotes that patients who underwent bridging anticoagulation had more bleeding events, which was statistically significant as compared to patients who did not undergo bridging. Also, there was no significant difference between thromboembolic events in both arms. The population studied in this meta-analysis only included patients with nonvalvular atrial fibrillation. Patients with mechanical heart valves, venous thromboembolism, and severe rheumatic heart disease were excluded. In their meta-analysis, Siegal et al ${ }^{6}$ derived the same conclusion, but the patient population was vastly different. They included patients with venous thromboembolism, mechanical heart valves, atrial fibrillation, and various other indications. In contrast, we included patients who were on anticoagulation primarily for non-valvular atrial fibrillation. Furthermore, our analysis only contains prospective studies. We also included in our analysis the landmark BRIDGE trial. ${ }^{11}$

An individualized approach is required when deciding whether or not to initiate peri-operative bridging. Mean $\mathrm{CHADS}_{2}$ score for the patients in the bridging and non-bridging arm (Supplementary Table 2, available online) was $<4$, which confers $<5 \%$ risk of thromboembolism per year (low risk), ${ }^{4}$ but in clinical practice there is a wide variation of $\mathrm{CHADS}_{2}$ scores encountered in patients with atrial fibrillation. Only $3 \%$ of participants had $\mathrm{CHADS}_{2}$ score of 5-6 in the BRIDGE trial; therefore, the result of this meta-analysis cannot be extrapolated to this subgroup. ${ }^{11}$ Patients with high $\mathrm{CHADS}_{2}$ score are usually the ones who benefit from bridging; ${ }^{4}$ hence, it is important to individualize the risk of bleeding and thromboembolism before committing or deferring bridging anticoagulation in a patient..$^{13}$ It is also difficult to extrapolate these results to the more contemporary $\mathrm{CHA}_{2} \mathrm{DS}_{2}$ Vasc scoring system, since most of the patients included in this meta-analysis were stratified using the $\mathrm{CHADS}_{2}$ score. Chao et $\mathrm{al}^{14}$ identified that patients with a $\mathrm{CHADS}_{2}$ score of 0 can have an annual stroke rate of $4.47 \%$ when stratified using the $\mathrm{CHA}_{2} \mathrm{DS}_{2}$ VASc score. Guidance can be taken from the 2017 American College of Cardiology guidelines on peri-operative management of anticoagulation in patients with non-valvular atrial fibrillation. ${ }^{4}$ Patients with a $\mathrm{CHA}_{2} \mathrm{DS}_{2} \mathrm{VASc}$ score of 1-4 were labelled as low risk, and bridging anticoagulation was not indicated among this subset.

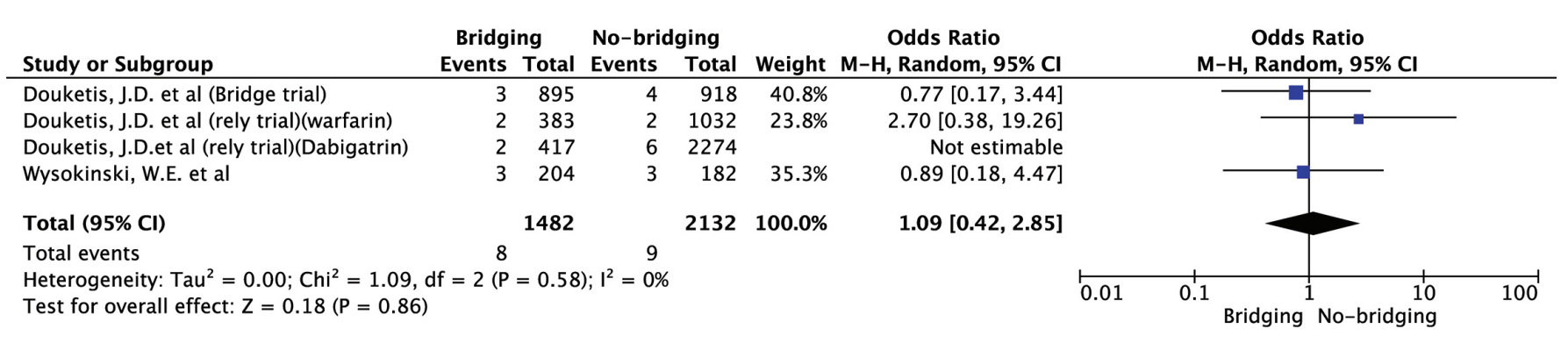

Figure 4. Forest plot for primary efficacy outcome (only warfarin) 


\begin{tabular}{|c|c|c|c|c|c|c|c|c|c|c|}
\hline Study or Subgroup & \multicolumn{2}{|c|}{ Bridging } & \multicolumn{2}{|c|}{ No-bridging } & Weight & $\begin{array}{l}\text { Odds Ratio } \\
\text { M-H, Random, } 95 \% \mathrm{Cl}\end{array}$ & \multicolumn{4}{|c|}{$\begin{array}{l}\text { Odds Ratio } \\
\text { M-H, Random, } 95 \% \mathrm{Cl}\end{array}$} \\
\hline Douketis, J.D. et al (Bridge trial) & 29 & 895 & 12 & 918 & $24.8 \%$ & $2.53[1.28,4.99]$ & & & $\longrightarrow$ & \\
\hline Douketis, J.D. et al (rely trial)(warfarin) & 26 & 383 & 16 & 1032 & $27.6 \%$ & $4.62[2.45,8.72]$ & & & & \\
\hline Douketis, J.D.et al (rely trial)(Dabigatrin) & 27 & 417 & 42 & 2274 & $39.5 \%$ & $3.68[2.24,6.04]$ & & & $\neg-$ & \\
\hline Wysokinski, W.E. et al & 6 & 204 & 4 & 182 & $8.2 \%$ & $1.35[0.37,4.86]$ & & & & \\
\hline Total $(95 \% \mathrm{Cl})$ & & 1899 & & 4406 & $100.0 \%$ & $3.29[2.25,4.81]$ & & & & \\
\hline Total events & 88 & & 74 & & & & & & & \\
\hline \multicolumn{7}{|c|}{$\begin{array}{l}\text { Heterogeneity: } \mathrm{Tau}^{2}=0.03 ; \mathrm{Chi}^{2}=3.76, \mathrm{df}=3(\mathrm{P}=0.29) ; \mathrm{I}^{2}=20 \% \\
\text { Test for overall effect: } \mathrm{Z}=6.15(\mathrm{P}<0.00001)\end{array}$} & 0.01 & 0.1 & 1 No-bridging & 100 \\
\hline
\end{tabular}

Figure 5. Forest plot for primary safety outcome

The decision of whether or not to bridge patients with a $\mathrm{CHADS}_{2} \mathrm{DS}_{2}$ VASc of $\geq 5$ was dependent on thrombotic and bleeding risk assessment of individual patients.

The studies in our analysis did not have patients who had recent venous thromboembolism or had mechanical valves, so the results are not applicable to this subset of patients who require more close monitoring and robust risk-benefit assessment. PERIOP2 (a double-blind randomized control trial of postoperative low molecular weight heparin bridging therapy versus placebo bridging therapy for patients who are at high risk for arterial thromboembolism) trial, once completed, will be able to provide more guidance in this subset of high-risk patients. ${ }^{15}$ Also, the studies in this analysis did not provide any guidance regarding the bridging anticoagulation at the time of initiation of oral anticoagulation (warfarin) in new-onset atrial fibrillation patients. The results could be extrapolated, but again, each decision must be personalized depending upon the risk factors profile of the patient. The study by Steinberg et al ${ }^{16}$ is congruent with our results, but it was not included in our analysis, as it did not meet criteria (it had patients with mechanical valves). ${ }^{16}$

NOACs are as effective as vitamin $\mathrm{K}$ antagonist for nonvalvular atrial fibrillation. ${ }^{17}$ Dabigatran was the first NOAC to be approved by the Food and Drug Administration (FDA) in 2010 after the Randomized Evaluation of Long-Term Anticoagulant Therapy (RELY) was published. ${ }^{18}$ Due to their pharmacokinetic properties, NOACs have a fast onset and offset of action, which does not usually require bridging therapy. ${ }^{13,18,19}$ In a subset of the RELY trial, bridging in patients taking dabigatran was associated with three times more major bleeding $(6.5 \%$ vs $1.8 \% \mathrm{OR}=3.68 ; 95 \% \mathrm{CI}$ : $2.24-6.04 ; P$ $<.001$ ) without any statistical difference in stroke. ${ }^{7}$ NOAC use for bridging instead of parenteral agents is thought-provoking, but some NOACs (factor Xa inhibitors) do alter the INR level; therefore, it could represent a false coagulation status prior to surgery when used for bridging in patients with atrial fibrillation. Furthermore, use of NOAC with vitamin K antagonist would theoretically increase the risk of bleeding, although the magnitude is unknown. ${ }^{20,21}$ The Perioperative Anticoagulant Use for Surgery Evaluation (PAUSE) study demonstrated low rates of major bleeding and arterial thromboembolism in patients with atrial fibrillation when NOAC therapy is interrupted without bridging in anticipation of an elective surgery. ${ }^{22}$ NOACs have a quick on and off action, which precludes the use of bridging therapy. ${ }^{13,20}$

The limitations of this meta-analysis are primarily due to the inherent limitations of the included studies. We were restricted to only three studies in this meta-analysis, due to lack of randomized and non-randomized trials on this topic. One study included in this review was non-randomized, introducing the possibility of selection and sample bias. Baseline characteristics, including baseline cardiac function and bleeding risk profile, were different as well, introducing heterogeneity. Finally, we restricted this study to PubMed, Medline (Ovid), and Cochrane databases; hence, it is possible there are other studies matching our inclusion criteria that were not included in our meta-analysis.

In conclusion, this meta-analysis provides insight regarding efficacy and safety of bridging anticoagulation during temporary

\begin{tabular}{|c|c|c|c|c|c|c|c|c|c|c|}
\hline Study or Subgroup & \multicolumn{2}{|c|}{ Bridging } & \multicolumn{2}{|c|}{ No-bridging } & Weight & $\begin{array}{c}\text { Odds Ratio } \\
\text { M-H, Random, } 95 \% \mathrm{Cl}\end{array}$ & \multicolumn{4}{|c|}{$\begin{array}{c}\text { Odds Ratio } \\
\text { M-H, Random, } 95 \% \mathrm{Cl}\end{array}$} \\
\hline Douketis, J.D. et al (Bridge trial) & 29 & 895 & 12 & 918 & $39.8 \%$ & $2.53[1.28,4.99]$ & & & $\longrightarrow$ & \\
\hline Douketis, J.D. et al (rely trial)(warfarin) & 26 & 383 & 16 & 1032 & $42.5 \%$ & $4.62[2.45,8.72]$ & & & & \\
\hline Douketis, J.D.et al (rely trial)(Dabigatrin) & 27 & 417 & 42 & 2274 & & Not estimable & & & & \\
\hline Wysokinski, W.E. et al & 6 & 204 & 4 & 182 & $17.7 \%$ & $1.35[0.37,4.86]$ & & & - & \\
\hline Total $(95 \% \mathrm{Cl})$ & & 1482 & & 2132 & $100.0 \%$ & $2.92[1.58,5.40]$ & & & & \\
\hline Total events & 61 & & 32 & & & & & & & \\
\hline \multicolumn{7}{|c|}{$\begin{array}{l}\text { Heterogeneity: } \mathrm{Tau}^{2}=0.13 ; \mathrm{Chi}^{2}=3.51, \mathrm{df}=2(\mathrm{P}=0.17) ; \mathrm{I}^{2}=43 \% \\
\text { Test for overall effect: } \mathrm{Z}=3.43(\mathrm{P}=0.0006)\end{array}$} & 0.01 & Bridging No-bridging & 1 No-bridging & 100 \\
\hline
\end{tabular}

Figure 6. Forest plot for primary safety outcome (only warfarin) 
interruption of OAC. The results of this meta-analysis are consistent with the individual studies, particularly the BRIDGE trial. There is more risk to bridging anticoagulation than benefit in patients with atrial fibrillation. Although the current evidence and this meta-analysis identified higher risk of major bleeding in patients who were provided bridging anticoagulation, the findings can only be applied after appropriate risk and benefit assessment. Patients with higher $\mathrm{CHADS}_{2}(\geq 5)$ score may have a higher risk of stroke than major bleeding; hence, perioperative bridging anticoagulation may be justified in this population as indicated in 2017 American College of Cardiology and 2012 American College of Chest Physicians guidelines. ${ }^{4,5}$ This manuscript is neither in favor of bridging anticoagulation or against it; however, it provides data that could help to make this important decision. The decision should always be individualized after contemplating the bleeding risk versus the thromboembolic risk.

\section{References}

1. Chugh SS, Havmoeller R, Narayanan K, et al. Worldwide epidemiology of atrial fibrillation: a Global Burden of Disease 2010 Study. Circulation. 2014;129(8):837-847.

2. Miyasaka Y, Barnes ME, Gersh BJ, et al. Secular trends in incidence of atrial fibrillation in Olmsted County, Minnesota, 1980 to 2000, and implications on the projections for future prevalence [published correction appears in Circulation. 2006 Sep 12;114(11):e498]. Circulation. 2006;114(2):119-125.

3. Spyropoulos AC, Turpie AG, Dunn AS, et al. Clinical outcomes with unfractionated heparin or low-molecularweight heparin as bridging therapy in patients on long-term oral anticoagulants: the REGIMEN registry. J Thromb Haemost. 2006;4(6):1246-1252.

4. Doherty JU, Gluckman TJ, Hucker WJ, et al. 2017 ACC Expert Consensus Decision Pathway for Periprocedural Management of Anticoagulation in Patients With Nonvalvular Atrial Fibrillation: A Report of the American College of Cardiology Clinical Expert Consensus Document Task Force. J Am Coll Cardiol. 2017;69(7):871-898.

5. Douketis JD, Spyropoulos AC, Spencer FA, et al. Perioperative management of antithrombotic therapy: Antithrombotic Therapy and Prevention of Thrombosis, 9th ed: American College of Chest Physicians EvidenceBased Clinical Practice Guidelines [published correction appears in Chest. 2012 Apr;141(4):1129]. Chest. 2012;141(2 Suppl):e326S-e350S.

6. Siegal D, Yudin J, Kaatz S, Douketis JD, Lim W, Spyropoulos AC. Periprocedural heparin bridging in patients receiving vitamin $\mathrm{K}$ antagonists: systematic review and meta-analysis of bleeding and thromboembolic rates. Circulation. 2012;126(13):16301639.
7. Douketis JD, Healey JS, Brueckmann M, et al. Perioperative bridging anticoagulation during dabigatran or warfarin interruption among patients who had an elective surgery or procedure. Substudy of the RE-LY trial. Thromb Haemost. 2015;113(3):625-632.

8. Moher D, Liberati A, Tetzlaff J, Altman DG; PRISMA Group. Preferred reporting items for systematic reviews and meta-analyses: the PRISMA statement. Ann Intern Med. 2009;151(4):264-W64.

9. Higgins JP, Altman DG, Gøtzsche PC, et al. The Cochrane Collaboration's tool for assessing risk of bias in randomised trials. BMJ. 2011;343:d5928. Published 2011 Oct 18

10. Turner RM, Davey J, Clarke MJ, Thompson SG, Higgins JP. Predicting the extent of heterogeneity in meta-analysis, using empirical data from the Cochrane Database of Systematic Reviews. Int J Epidemiol. 2012;41(3):818-827.

11. Douketis JD, Spyropoulos AC, Kaatz S, et al. Perioperative Bridging Anticoagulation in Patients with Atrial Fibrillation. N Engl J Med. 2015;373(9):823-833.

12. Wysokinski WE, McBane RD, Daniels PR, et al. Periprocedural anticoagulation management of patients with nonvalvular atrial fibrillation [published correction appears in Mayo Clin Proc. 2008 Jul;83(7):851]. Mayo Clin Proc. 2008;83(6):639-645.

13. January CT, Wann LS, Alpert JS, et al. 2014 AHA/ ACC/HRS guideline for the management of patients with atrial fibrillation: a report of the American College of Cardiology/American Heart Association Task Force on Practice Guidelines and the Heart Rhythm Society [published correction appears in J Am Coll Cardiol. 2014 Dec 2;64(21):2305-7]. J Am Coll Cardiol. 2014;64(21):e1-e76.

14. Chao TF, Liu CJ, Tuan TC, et al. Comparisons of CHADS2 and CHA2DS2-VASc scores for stroke risk stratification in atrial fibrillation: Which scoring system should be used for Asians?. Heart Rhythm. 2016;13(1):46-53.

15. Rechenmacher SJ, Fang JC. Bridging Anticoagulation: Primum Non Nocere. J Am Coll Cardiol. 2015;66(12):1392-1403.

16. Steinberg BA, Peterson ED, Kim S, et al. Use and outcomes associated with bridging during anticoagulation interruptions in patients with atrial fibrillation: findings from the Outcomes Registry for Better Informed Treatment of Atrial Fibrillation (ORBIT-AF). Circulation. 2015;131(5):488-494.

17. Levy JH, Douketis J, Weitz JI. Reversal agents for non-vitamin K antagonist oral anticoagulants. Nat Rev Cardiol. 2018;15(5):273-281. 
18. Mekaj YH, Mekaj AY, Duci SB, Miftari EI. New oral anticoagulants: their advantages and disadvantages compared with vitamin $\mathrm{K}$ antagonists in the prevention and treatment of patients with thromboembolic events. Ther Clin Risk Manag. 2015;11:967-977. Published 2015 Jun 24.

19. Verma A, Cairns JA, Mitchell LB, et al. 2014 focused update of the Canadian Cardiovascular Society Guidelines for the management of atrial fibrillation [published correction appears in Can J Cardiol. 2014 Dec;30(12):1495] [published correction appears in Can J Cardiol. 2015 Oct;31(10):1302]. Can J Cardiol. 2014;30(10):1114-1130.

20. Pollack CV Jr. Coagulation assessment with the new generation of oral anticoagulants. Emerg Med J. 2016;33(6):423-430.

21. Garwood CL, Korkis B, Grande D, Hanni C, Morin A, Moser LR. Anticoagulation Bridge Therapy in Patients with Atrial Fibrillation: Recent Updates Providing a Rebalance of Risk and Benefit. Pharmacotherapy. 2017;37(6):712-724.

22. Douketis JD, Spyropoulos AC, Duncan J, et al. Perioperative Management of Patients With Atrial Fibrillation Receiving a Direct Oral Anticoagulant [published online ahead of print, 2019 Aug 5]. JAMA Intern Med. 2019;179(11):1469-1478.

\section{Author Affiliations}

Muhammad Umer Siddiqui, MD ${ }^{*, 1}$; Ahmed K. Pasha, MDץ, ; Ibtisam Rauf, MDћ; Justin Z. Lee, MD§; Muhammad Danial Siddiqui, MD\|; Youssef Yaacoub, MDף; Reza Movahed, MD, FACC, PhD\#

*Marshfield Clinic Health System, Eau Claire, Wisconsin, USA [Current affiliation: George Washington University, Washington, DC, USA

†Mayo Clinic Health System, Rochester, Minnesota, USA +Northwestern University Feinberg School of Medicine, Department of Physical Therapy and Human Movement, Chicago, Illinois, USA

§Mayo Clinic Arizona, Phoenix, Arizona, USA

॥University of South Dakota, Vermillion, South Dakota, USA

$\checkmark$ Catholic Medical Center, Manchester, New Hampshire, USA

\#University of Arizona Health Sciences, Tucson, Arizona, USA

${ }^{1}$ both authors contributed equally to this work 